\title{
BMJ Open Economic analysis of endovascular drug-eluting treatments for femoropopliteal artery disease in the UK
}

\author{
Konstantinos Katsanos, ${ }^{1}$ Benjamin P Geisler, ${ }^{2,3}$ Abigail M Garner, ${ }^{2}$ Hany Zayed, ${ }^{1}$ \\ Trevor Cleveland, ${ }^{4}$ Jan B Pietzsch ${ }^{2}$
}

To cite: Katsanos K Geisler BP, Garner AM, et al. Economic analysis of endovascular drug-eluting treatments for femoropopliteal artery disease

in the UK. BMJ Open 2016;6: e011245. doi:10.1136/ bmjopen-2016-011245

- Prepublication history and additional material is available. To view please visit the journal (http://dx.doi.org/ 10.1136/bmjopen-2016011245).

Received 23 January 2016 Revised 8 March 2016 Accepted 6 April 2016

CrossMark

${ }^{1}$ Guy's and St. Thomas' NHS Foundation Trust, London, UK

${ }^{2}$ Wing Tech Inc., Menlo Park, California, USA

${ }^{3}$ Massachusetts General Hospital/Harvard Medical School, Boston,

Massachusetts, USA

${ }^{4}$ Sheffield Teaching Hospitals NHS Foundation Trust, Sheffield, UK

Correspondence to Dr Konstantinos Katsanos; katsanos@med.upatras.gr \& konstantinos.katsanos@kcl. ac.uk

\section{ABSTRACT}

Objectives: To estimate the clinical and economic impact of drug-eluting endovascular treatment strategies for femoropopliteal artery disease compared with current standard of care.

Design: Systematic literature search to pool target lesion revascularisations (TLR). Model-based perpatient cost impact and quasi-cost-effectiveness projection over 24 months based on pooled TLRs and current reimbursement.

Setting: The UK's National Health Service (NHS). Participants: Patients presenting with symptomatic femoropopliteal disease eligible for endovascular treatment.

Interventions: Current National Institute for Health and Care Excellence (NICE) guideline-recommended treatment with percutaneous transluminal balloon angioplasty (PTA) and bailout bare metal stenting (BMS) versus primary BMS placement, or drug-coated balloon (DCB), or drug-eluting stent (DES) treatment.

Primary and secondary outcome measures: 24month per-patient cost impact to NHS (primary outcome). Secondary outcomes: pooled 24-month TLR rates; numbers needed to treat (NNTs); cost per TLR avoided and estimated incremental cost-effectiveness ratio (ICER) in £ per quality-adjusted life year (QALY).

Results: $\mathrm{N}=28$ studies were identified, reporting on 5167 femoropopliteal lesions. Over 24 months, DCB, DES and BMS reduced TLRs of de novo lesions from $36.2 \%$ to $17.6 \%, 19.4 \%$ and $26.9 \%$, respectively, at an increased cost of $£ 43, £ 44$ and $£ 112$. NNTs to avoid 1 TLR in 24 months were 5.4, 6.0 and 10.8, resulting in cost per TLR avoided of £231, £264 and £1204. DCB was estimated to add 0.011 QALYS, DES 0.010 QALYS and BMS 0.005 QALYs, resulting in estimated ICERs of $£ 3983$, £4534 and £20 719 per QALY gained. A subset analysis revealed more favourable clinical and economic outcomes for a $3.5 \mu \mathrm{g} / \mathrm{mm}^{2} \mathrm{DCB}$ with urea excipient, compared with the rest of DCBs. A modest reduction of $10 \%$ in DCB and DES prices made drugeluting treatments dominant.

Conclusions: Widespread adoption of drug-eluting endovascular therapies for femoropopliteal disease would add meaningful clinical benefit at reasonable additional costs to the NHS. Based on currently

\section{Strengths and limitations of this study}

- To the best of our knowledge, this is the largest systematic literature review of different endovascular options for the femoropopliteal artery conducted to date (28 clinical studies were pooled reporting on more than 5000 femoropopliteal artery lesions) to inform a health economic analysis comparing percutaneous transluminal balloon angioplasty (PTA) and bailout bare metal stenting (BMS), or primary BMS placement, or drug-coated balloon application, or drug-eluting stent placement.

- The primary strength of the present study was the development of a robust decision-analytic per-patient cost impact model for the UK National Health Service (NHS) on the basis of the pooled probabilities of future target lesion revascularisation, whose results question the National Institute for Health and Care Excellence-guideline recommended standard of care (PTA and bailout BMS) and support a paradigm shift towards drug-eluting therapies.

- We explored several outcome measures, including the per-patient cost impact of drug-eluting therapies, the numbers needed to treat and the estimated incremental cost-effectiveness ratio of competing treatment strategies as measures of clinical effectiveness and cost-effectiveness, along with extensive subset and sensitivity analyses.

- The main limitation of the present health economic analysis is that it has been developed according to the current practice pattern, the market forces and tariff system applicable to the NHS in the UK.

- Our economic analysis is also limited by a 24-month time horizon in the absence of longer term data, and also by the potential underrepresentation of critical limb ischaemia cases.

available data, DCBs offer the highest clinical and economic value. 


\section{INTRODUCTION}

Peripheral artery disease causes significant morbidity and reduced quality of life for patients, with vascular restenosis and vessel failure leading to frequent revascularisation in some patients or even amputations, representing a significant economic burden on the UK National Health Service (NHS). ${ }^{1}{ }^{2}$ Current guidance from the National Institute for Health and Care Excellence (NICE) recommends offering percutaneous transluminal balloon angioplasty (PTA) only after advice on risk factor modification has been reinforced, a supervised exercise programme has been tried and imaging has confirmed a lesion suitable for intervention. ${ }^{3} 4$ Stenting is currently not recommended as a primary treatment for femoropopliteal disease. If stenting is needed or offered, the NICE guidance specifies that bare metal stents (BMS) should be used. Both drug-eluting stents (DESs) and drug-coated balloons (DCBs) have shown promising results in the femoropopliteal segment by reducing vascular restenosis and consequently the need for target lesion revascularisation (TLR). ${ }^{5}$ DCB in particular, as a relatively novel and effective treatment approach that does not require any long-term device implant, has not yet been considered in the current NICE guidance.

The objectives of the present study were (1) to systematically search for and synthesise the clinical efficacy of different endovascular treatment approaches taking into account the most recent published evidence on PTA, BMS, DCB and DES; (2) to estimate the per-patient cost impact on the NHS of these competing endovascular treatment strategies and (3) to study related secondary outcome measures of cost-effectiveness.

\section{MATERIALS AND METHODS}

We conducted a systematic literature search for clinical trials and registries reporting TLR rates in de novo superficial femoral artery (SFA) and/or popliteal artery disease (see online supplementary appendix). Treatment-specific probabilities of TLR were pooled and used in the budget impact decision-analytic model in order to estimate treatment-specific total reimbursement related to index procedure and applicable revascularisation over a 24-month time horizon. We pursued a primary analysis that assumed any repeat interventions to be treated according to the current guideline-recommended approach of PTA with bailout BMS, and a secondary analysis exploring variations in repeat revascularisation strategies.

The primary outcome was the 24-month per-patient cost impact to NHS. Secondary outcomes included pooled 24-month TLR rates; numbers needed to treat (NNTs); cost per TLR avoided and estimated incremental cost-effectiveness ratio (ICER) in $£$ per quality-adjusted life year (QALY).

\section{Systematic literature search and pooling}

Our systematic literature search was carried out in June 2015, and was performed as an update of prior PubMed and EMBASE searches completed in December 2012 for a budget impact analysis focused on the USA and Germany. ${ }^{6}$ In short, our search identified all relevant studies of endovascular interventions for treatment of de novo SFA lesions, limiting the analysis to one of the following four therapies-PTA, BMS, DES or DCB. Only studies that reported TLR as an end point were included. In order to avoid potential confounders and to ensure an unbiased comparison, we excluded studies reporting mean lesion lengths $>20 \mathrm{~mm}$, more complex lesions and studies that primarily investigated interventions of restenotic lesions.

Applying the same approach as in the prior analysis, we extracted the probability of TLR reported for the longest follow-up (up to 24 months) for each of the identified studies. ${ }^{6}$ Estimates of 24-month TLR probabilities of each of the four interventions were subsequently computed using a weighted pooling approach based on sample size. For studies only reporting shorter follow-up of 12 months, the 12-month TLR rates were pooled, and then extrapolated to the corresponding 24-month TLR probability, under the assumption of a constant hazard rate.

\section{Decision-analytic model structure and reintervention strategies}

A decision-analytic model was developed to estimate, by index procedure strategy, the primary and secondary end points of this analysis. The model considered the index procedure and up to one reintervention, over a 24-month analysis horizon. This time horizon was chosen as it reflects the follow-up horizon available for most of the included studies. Reintervention rates were based on the pooled rates of the four modalities PTA, BMS, DCB and DES. For strategies that included bailout stenting (ie, stenting performed if index balloon intervention was technically not feasible or suboptimal), the 24-month TLR was computed considering the primary device's TLR estimate for the cases not requiring bailout stenting, and the BMS-specific TLR for the remainder. Mortality was not considered given the limited time horizon of the analysis, and because the included clinical studies do not suggest a mortality difference related to the study devices or procedures.

The primary analysis compared BMS, DCB and DES strategies to current guideline-recommended approach. For this analysis, we assumed that any necessary reintervention would be performed using the same guideline-recommended approach (PTA with bailout BMS stenting), with an assumed $20 \%$ bailout proportion, based on recent data ${ }^{7-11}$ and our own clinical experience (coauthors KK, HZ, TC). Several additional treatment strategies, such as the use of drug-eluting therapies in case of a TLR, were considered in the secondary analysis to explore possible variations in reintervention approaches.

\section{Cost assumptions}

Costs were assumed based on the current 2015/2016 NHS England Tariff (Enhanced Tariff Option), Hospital 
Episodes Statistics (HES) data, and on market research data on current device prices of BMS, DCB and DES. The total 24-month costs calculated in this analysis included the reimbursement for the index procedure and applicable reintervention. Costs for each intervention were based on the complication-adjusted and market forces factor-adjusted inpatient tariff, as well as one outpatient specialist visit preintervention and postintervention, and the tariff for one colour duplex imaging (see table 1). For BMS, DCB and DES, the respective current selling price of the device was added, per current tariff rules which allow separate reimbursement as excluded devices.

\section{Computation of study end points}

The study end points were computed as follows: 24-month TLR was computed based on the pooled TLR rates, and where applicable, by considering the probability of bailout BMS stenting. Cost difference was computed considering the 24-month total cost of each strategy, versus the current guideline-recommended treatment strategy of PTA with bailout BMS stenting. NNTs were computed based on the absolute difference between estimated TLR rates. For each of the alternative strategies, cost difference and NNTs were multiplied to obtain cost per TLR avoided.
To obtain an estimate for incremental costeffectiveness, we considered a decrement of 0.06 QALYs associated with each TLR on the basis of previously published health utilities. ${ }^{13}$ This decrement was based on the observed differences in health-related quality of life (utility) between pretreatment baseline and posttreatment, and also based on the differences in health-related quality of life observed between patients requiring a TLR and patients not requiring a TLR. ${ }^{13}$ Estimated QALY gain was computed by multiplying the difference in TLR rate by the QALY decrement, under the assumption of no mortality difference. The resulting ICER estimate is the ratio of incremental costs to incremental QALYs. For all computations, we opted to not discount costs or effects because of the short follow-up horizon of the analysis of only 24 months and the fact that most costs are incurred at time zero.

\section{Base cases, subset analyses and sensitivity analyses}

The base cases of the primary and secondary analysis, as described earlier, reflect point estimates of the 24-month cost per patient to NHS by index procedure strategy, as well as secondary outcome measures including cost per TLR avoided, NNT to avoid one TLR in 24 months, estimated QALY gain and estimated ICER. A subset analysis was computed to estimate the differences

Table 1 Input parameter assumptions used in the model

\begin{tabular}{|c|c|c|}
\hline Variable & Definition & Source \\
\hline \multicolumn{3}{|l|}{ Clinical parameters } \\
\hline \multicolumn{3}{|l|}{ Mean 24-month proportion of TLRs } \\
\hline PTA & $38.5 \%$ & Weighted pooling of TLRs in identified trials (see online \\
\hline BMS & $26.9 \%$ & supplementary table) \\
\hline DES & $19.4 \%$ & \\
\hline DCB & $17.6 \%$ & \\
\hline DCB (paclitaxel with urea excipient-In.Pact) & $11.2 \%$ & \\
\hline DCB (other) & $21.9 \%$ & \\
\hline \multicolumn{3}{|l|}{ Probability of bailout stenting in balloon procedures } \\
\hline Probability of bailout stenting & 0.20 & $\begin{array}{l}\text { Author estimate based on Schillinger }{ }^{12} \\
\text { and Laird et } \mathrm{Il}^{7}\end{array}$ \\
\hline \multicolumn{3}{|l|}{ Cost parameters } \\
\hline \multicolumn{3}{|l|}{ Costs associated with every intervention } \\
\hline $\begin{array}{l}\text { HRG best practice tariff, therapeutic endovascular } \\
\text { interventions, CC-adjusted and MFF-adjusted }\end{array}$ & $£ 1702$ & Based on HRG QZ15B, QZ15B, adjusted for frequency \\
\hline $\begin{array}{l}\text { Outpatient tariff for specialist visit preintervention } \\
\text { and postintervention, including colour duplex } \\
\text { imaging }\end{array}$ & $£ 323$ & WF01B (1xpre, 1xpost), HRG RA24Z \\
\hline \multicolumn{3}{|l|}{ Data used to compute HRG tariff } \\
\hline Percentage of intermediate $\mathrm{CC}$ cases & $59 \%$ & Based on $2014 / 2015$ admissions data \\
\hline Percentage of non-CC cases & $41 \%$ & \\
\hline MFF & 1.0809 & Average MFF for the NHS England \\
\hline \multicolumn{3}{|l|}{ Device cost (added to respective procedure cost) } \\
\hline BMS & $£ 384$ & Average annual selling prices from Decision Resources \\
\hline DES & $£ 474$ & Group Reports (US PV Pricetrack, EU PV Marketrack) \\
\hline $\mathrm{DCB}$ & $£ 512$ & through September 2015 \\
\hline DCB (paclitaxel with urea excipient-In.Pact) & $£ 636$ & \\
\hline
\end{tabular}

BMS, bare metal stents; CC, Complication and Comorbidity; DCB, drug-coated balloon; DES, drug-eluting stent; HRG, Healthcare Resource Group; MFF, market forces factor; NHS, National Health Service; PTA, percutaneous transluminal balloon angioplasty; TLR, target lesion revascularisation. 
between $3.5 \mu \mathrm{g} / \mathrm{mm}^{2}$ urea excipient-based DCB (IN. PACT Admiral, Medtronic) and other DCBs, as there is experimental evidence $^{14}{ }^{15}$ which suggests different DCB coating formulations with different pharmacokinetic profiles may produce different vessel tissue bioavailability and ultimately result in significant differences of DCB clinical results. ${ }^{7}{ }^{16}$ Several sensitivity analyses were performed to study the effect of parameter uncertainty on the base case and subset analysis results. These included an assumed device use of 1.5 instead of 1 device per procedure for DCB and DES strategies to account for longer lesions, a decrease in assumed device costs for BMS, DES and DCB by $20 \%$, an increase in device costs by $10 \%$, and a scenario in which DCB and DES costs were reduced by $10 \%$. In addition, we studied the effect of parameter uncertainty in each therapy's clinical effectiveness by considering lower and upper TLR bounds. To obtain realistic TLR ranges for sensitivity analysis, we pooled the subsets of higher and lower performing studies separately for each therapy (see online supplementary appendix), yielding 24-month TLR rate ranges of $30.8-47.0 \%$ for PTA, $21.9-33.1 \%$ for BMS, $11.8-23.8 \%$ for DCB and $17.5-36.0 \%$ for DES.

\section{RESULTS}

\section{Systematic literature search}

We identified a total of 32 publications representing 28 studies or registries published between 2006 and 2015 that fulfilled our criteria. ${ }^{4} 7-9$ 11 $17-43$ The Preferred Reporting Items for Systematic Reviews and Meta-Analyses diagram outlining the results of the systematic search process is included in the online supplementary appendix. The total number of lesions included was 5167 ( $\mathrm{n}=886$ for PTA, 2463 for BMS, 989 for DES and 829 for DCB, with lesion counts based on numbers reported at longest follow-up). Most patients suffered from intermittent claudication (Rutherford class 2 or 3), and $15-20 \%$ presented with critical limb ischaemia (CLI). Lesion location was primarily in the SFA.

\section{Clinical efficacy}

The pooled 24-month TLR estimate for PTA was 38.5\% (based on a total of 13 studies). For BMS, the TLR estimate was $26.9 \%$ (15 studies), and for DES $19.4 \%$ (4 studies). The pooled TLR estimate for DCB was $17.6 \%$ (nine studies). Subset analyses for DCB yielded TLR estimates of $11.2 \%$ for DCB with urea excipient (IN.PACT Admiral; three studies), ${ }^{7} 2340$ and $21.9 \%$ for all other DCB (six studies). ${ }^{17} 18$ 24-26 41 A detailed overview of the studies and the TLR rate computations from reported 12-month and 24-month follow-up are shown in table 2.

\section{Economic analysis results}

The average per-patient cost over a 24-month period, including the index procedure and applicable reintervention costs, was lowest for the current guideline-recommended strategy, PTA with bailout BMS stenting, at £2863, followed by DCB (incremental £43), DES (incremental £44) and primary BMS (incremental $£ 112)$. The projected cost increases of drug-eluting therapies of $<2 \%$ were accompanied by marked reductions in TLRs, with DCB reducing the 24-month reintervention rate to $17.6 \%$, and DES to $19.4 \%$ compared with $36.2 \%$ in case of the guideline-recommended strategy. Primary BMS placement, at increased cost of $3.9 \%$, was projected to be associated with $26.9 \%$ TLRs at 24 months (see figure 1).

The NNT was lowest for DCB (5.4), followed by DES (6.0) and BMS (10.8). Resulting costs per TLR avoided were £231 and £264 for DCB and DES, respectively, and $£ 1204$ for BMS. Estimated QALY gains were 0.005 for BMS 0.010 for DES and 0.011 for DCB, resulting in the estimated ICERs of $£ 3983$ (DCB), £4534 (DES) and $£ 20719$ (BMS) per QALY gained. See table 3 for details.

\section{Subset and sensitivity analyses}

The performed subset analyses comparing the study outcomes of $3.5 \mu \mathrm{g} / \mathrm{mm}^{2}$ urea excipient-based DCBs (Medtronic IN.PACT) to those of the remainder of DCBs showed lower TLR rates for the urea-based devices (11.2\%, compared with $21.9 \%$ ), resulting in lower NNTs (4.0 instead of 7.0). Cost per TLR avoided and the estimated ICER were lower with the $3.5 \mu \mathrm{g} / \mathrm{mm}^{2}$ urea excipient-based DCBs (£31 vs £947 and £2259 vs £16 290 per QALY gained, respectively).

The conducted sensitivity analyses of the primary, base case, analysis tested the effect of using more than one device, and of variations in device prices. Under the assumption of 1.5 devices per procedure used for the DCB and DES strategies, a higher cost difference of $£ 299$ and £281 was found, resulting in increased cost per TLR avoided of $£ 1604$ and $£ 1675$, and ICERs of $£ 27596$ and £28 815 per QALY gained for DCB and DES, respectively. Urea excipient-based DCBs had more favourable results than the non-urea-based DCBs in these scenarios as well (see online supplementary appendix). For the multiple device use scenarios, DCB and DES were the economically less favourable strategies, as compared with BMS.

In case of variations in the prices of DES and DCB, a reduction in device prices by $10 \%$ would make DCB and DES economically dominant, providing improved clinical outcomes at overall cost savings for the NHS budget at 2 years follow-up ( $£ 8$ saving for DCB, $£ 3$ saving for DES). See details in the online supplementary appendix.

Lower clinical effectiveness of PTA (24-month TLR of $47.0 \%$ ) led to more favourable outcomes for BMS, DCB and DES, making all three strategies cost saving and thus dominant, while higher PTA performance (30.8\%) increased added cost of BMS, DCB and DES to £242, $£ 173$ and $£ 174$, respectively. Lower BMS performance (TLR 33.1\%) increased added cost of the BMS first strategy to £216, but decreased DCB and DES added cost to 
Table 2 Total pooled 24-month TLR probabilities based on identified studies for therapies PTA, BMS, DCB and DES

\begin{tabular}{|c|c|c|c|c|c|c|c|c|}
\hline Therapy & Study & $\begin{array}{l}\text { 12-Month } \\
\text { reported TLR }\end{array}$ & $\begin{array}{l}\text { Reported } \\
\text { lesions (n) }\end{array}$ & $\begin{array}{l}\text { Pooled } \\
\text { 12-month TLR }\end{array}$ & $\begin{array}{l}\text { 24-Month } \\
\text { reported TLR }\end{array}$ & $\begin{array}{l}\text { Reported } \\
\text { lesions (n) }\end{array}$ & $\begin{array}{l}\text { Pooled } \\
\text { 24-month TLR }\end{array}$ & $\begin{array}{l}\text { Total pooled } \\
\text { 24-month TLR } \\
\text { estimate (\%) }\end{array}$ \\
\hline \multirow[t]{11}{*}{$\overline{\text { PTA }}$} & $\mathrm{FAST}^{20}$ & $18.3 \%$ & 115 & $19.5 \%$ & \multirow{6}{*}{\multicolumn{3}{|c|}{$\begin{array}{l}\text { Estimation of 24-month TLR based on } \\
\text { constant hazard rate assumption }\end{array}$}} & \multirow{11}{*}{38.5} \\
\hline & ZILVER-PTX ${ }^{21}$ & $17.5 \%$ & 223 & & & & & \\
\hline & PACIFIER ${ }^{23}$ & $27.9 \%$ & 43 & & & & & \\
\hline & BIOLUX-P| ${ }^{24}$ & $41.7 \%$ & 24 & & & & & \\
\hline & LEVANT $2^{26}$ & $16.8 \%$ & 143 & & & & & \\
\hline & COMPLIANCE $360^{27}$ & $21.7 \%$ & 23 & & & & & \\
\hline & THUNDER $^{17}$ & & & & $51.9 \%$ & 54 & $44.7 \%$ & \\
\hline & FEM-PAC ${ }^{18}$ & & & & $50.0 \%$ & 42 & & \\
\hline & RESILIENT $^{8} 19$ & & & & $58.2 \%$ & 72 & & \\
\hline & LEVANT $1^{25}$ & & & & $48.8 \%$ & 41 & & \\
\hline & IN.PACT SFA ${ }^{117}$ & & & & $28.3 \%$ & 106 & & \\
\hline \multirow[t]{15}{*}{ BMS } & $\mathrm{FAST}^{20}$ & $14.9 \%$ & 114 & $15.3 \%$ & \multirow{11}{*}{\multicolumn{3}{|c|}{$\begin{array}{l}\text { Estimation of 24-month TLR based on } \\
\text { constant hazard rate assumption }\end{array}$}} & \multirow[t]{15}{*}{26.9} \\
\hline & DURABILITY I ${ }^{4}$ & $20.9 \%$ & 134 & & & & & \\
\hline & Diehl et $a^{29}$ & $17.4 \%$ & 46 & & & & & \\
\hline & COBRA $^{30}$ & $16.0 \%$ & 43 & & & & & \\
\hline & ETAP $^{32}$ & $14.7 \%$ & 99 & & & & & \\
\hline & DURABILITY $\|^{33}$ & $14.0 \%$ & 287 & & & & & \\
\hline & $\mathrm{EPIC}^{34}$ & $7.7 \%$ & 88 & & & & & \\
\hline & Gabriella et $a^{\beta 5}$ & $26.8 \%$ & 68 & & & & & \\
\hline & SUPERB ${ }^{36}$ & $11.1 \%$ & 235 & & & & & \\
\hline & MARIS $^{37}$ & $17.2 \%$ & 789 & & & & & \\
\hline & COMPLETE SE ${ }^{9}$ & $9.4 \%$ & 191 & & & & & \\
\hline & $\mathrm{SIROCCO}^{28}$ & & & & $13.3 \%$ & 46 & $19.8 \%$ & \\
\hline & RESILIENT $^{819}$ & & & & $22.2 \%$ & 134 & & \\
\hline & MISAGO-2 subc ${ }^{31}$ & & & & $15.1 \%$ & 41 & & \\
\hline & Leipzig SUPERA $500^{38}$ & & & & $21.0 \%$ & 148 & & \\
\hline \multirow[t]{9}{*}{$\mathrm{DCB}$} & PACIFIER ${ }^{23}$ & $7.1 \%$ & 42 & $11.9 \%$ & \multirow{3}{*}{\multicolumn{3}{|c|}{$\begin{array}{l}\text { Estimation of 24-month TLR based on } \\
\text { constant hazard rate assumption }\end{array}$}} & \multirow[t]{9}{*}{17.6} \\
\hline & BIOLUX-PI ${ }^{24}$ & $15.4 \%$ & 25 & & & & & \\
\hline & LEVANT $2^{26}$ & $12.3 \%$ & 285 & & & & & \\
\hline & THUNDER $^{17}$ & & & & $15.0 \%$ & 47 & $14.0 \%$ & \\
\hline & FEM-PAC ${ }^{18}$ & & & & $13.0 \%$ & 45 & & \\
\hline & IT-Registry ${ }^{40}$ & & & & $14.3 \%$ & 98 & & \\
\hline & LEVANT $1^{25}$ & & & & $35.7 \%$ & 42 & & \\
\hline & ILLUMENATE FIH ${ }^{41}$ & & & & $14.9 \%$ & 47 & & \\
\hline & IN.PACT SFA $^{117} 7$ & & & & $9.1 \%$ & 198 & & \\
\hline \multirow[t]{4}{*}{ DES } & STRIDES ${ }^{43}$ & $20.0 \%$ & 103 & $20.0 \%$ & \multicolumn{3}{|c|}{ Estimation of 24-month TLR } & \multirow[t]{4}{*}{19.4} \\
\hline & ZILVER-PTX ${ }^{21}$ & & & & $14.4 \%$ & 224 & $17.5 \%$ & \\
\hline & ZILVER-PTX SAS ${ }^{21}$ & & & & $19.5 \%$ & 615 & & \\
\hline & SIROCCO 28 & & & & $6.0 \%$ & 47 & & \\
\hline
\end{tabular}




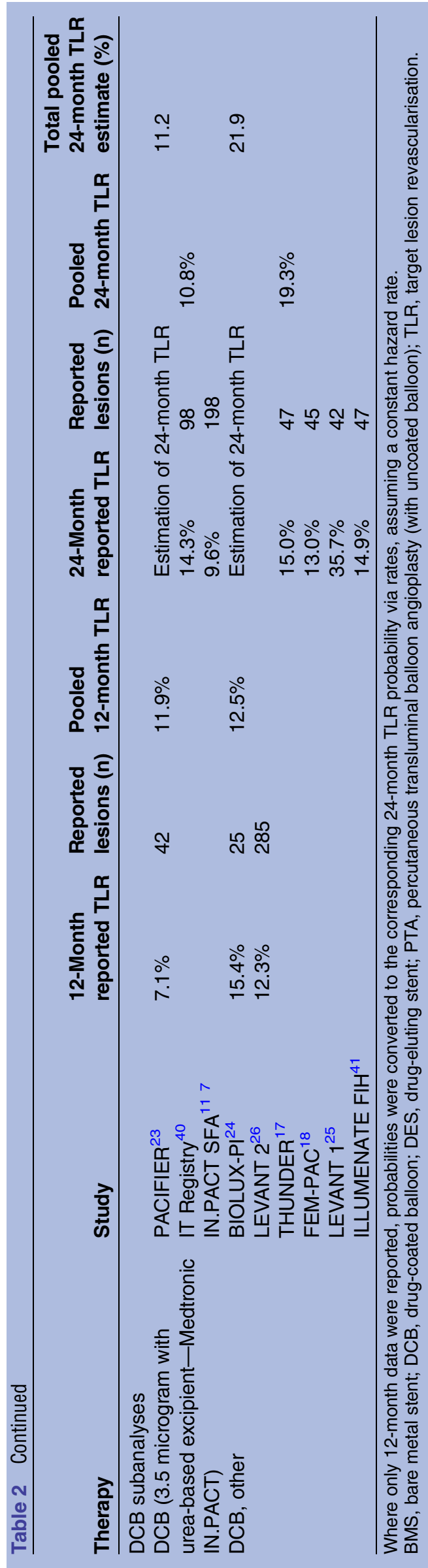

$£ 17$ and £18. Higher BMS performance (TLR 21.9\%) reduced its added cost to $£ 27$, but left DCB and DES the clinically preferred options. An assumed higher TLR rate of $23.8 \%$ for DCB increased the added cost of DCB to $£ 174$ and the ICER to $£ 24148$ per QALY gained, while a lower assumed TLR of $11.8 \%$ rendered DCB both cost saving and clinically superior. The low performance assumption of DES (TLR of $36.0 \%$ ) increased added cost to £393, at minimally improved clinical outcome compared with standard of care. Reducing the 24-month TLR of DES to $17.5 \%$ reduced added cost to $£ 4$ and made DES preferable to DCB in terms of costs and TLR performance. See online supplementary appendix for further detail.

Assessing, in our secondary analysis, various alternative scenarios for repeat intervention device usage for each of the considered index procedure strategies led directionally to similar findings (see table 4 and see online supplementary appendix). The lowest overall cost increases were £29 for DES (DES as index procedure, followed by PTA intervention) and $£ 30$ for DCB (DCB as index procedure, followed by PTA intervention). The highest cost increase of 2259 was found to be associated with a DCB with bailout BMS strategy pursued both for index and repeat intervention.

\section{DISCUSSION}

Current guidelines recommend an endovascular-first approach for the majority of femoropopliteal stenoses or occlusions. Although vein bypass surgery still has a role in case of long or heavily calcified chronic total occlusions and in patients with a favourable life expectancy, it is associated with significant perioperative morbidity, which has led many centres to adopt an endovascularfirst approach. ${ }^{3}{ }^{44-47}$ Although PTA and bailout stent placement has been long considered the endovascular standard of care, our results clearly indicate that drug-eluting endovascular therapies of femoropopliteal disease are associated with lower reintervention rates and marginally improved quality of life. We found DCB and DES to be associated with substantially lower TLR rates of around $18 \%$ and $19 \%$ over 24 months, compared with around $27 \%$ with a primary BMS strategy and around $36 \%$ with current standard of care as per NICE guidance. In addition, compared with standard of care, we found single-digit NNTs of 5 in case of a DCB-first treatment and 6 in case of a DES-first treatment, highlighting the high efficacy of drug-eluting technologies in improving clinical outcomes of the femoropopliteal artery.

Most interestingly, these improved clinical outcomes were found to be associated with limited increases in overall cost for the NHS budget. For the base case scenario assuming current tariffs and market forces, drug-eluting treatments resulted in an incremental budget impact of only £43-£44, or around $2 \%$, per patient at 24 months, with a favourable projected ICER 


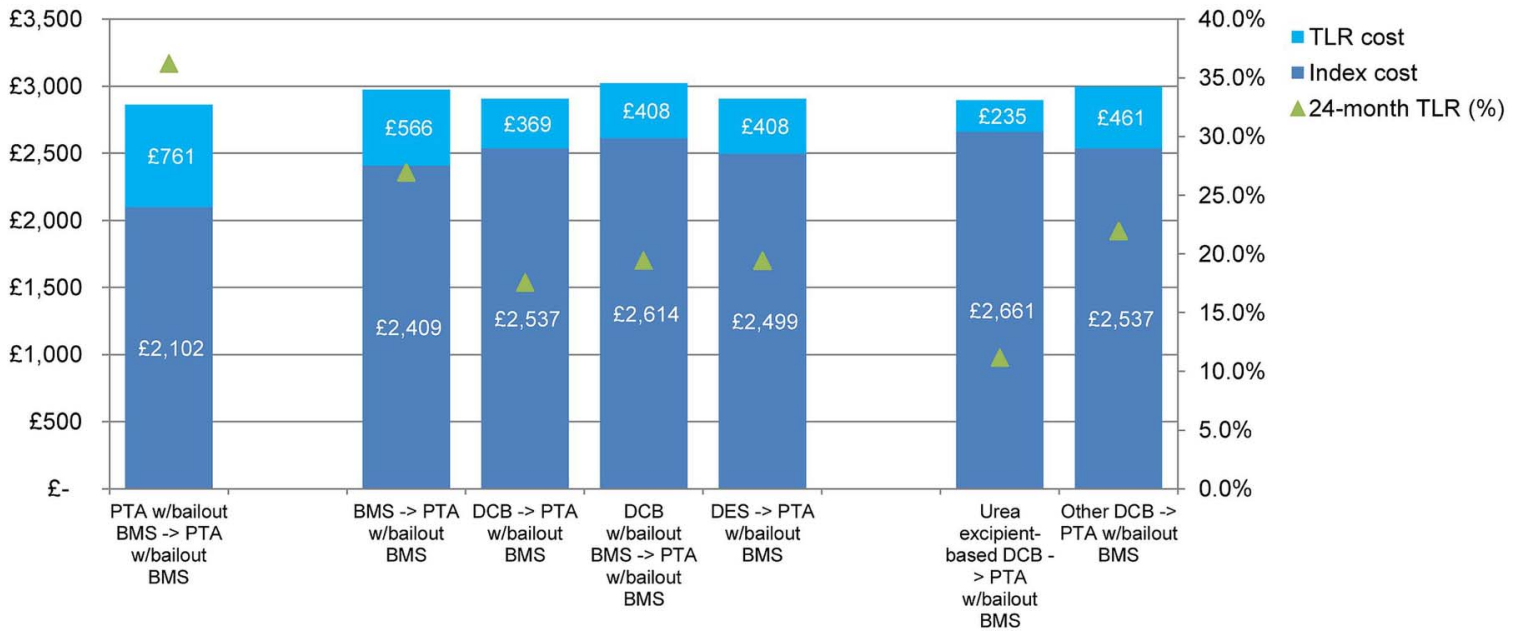

Figure 1 Twenty-four-month cost, by index and reintervention procedure, and 24-month TLR projection for the primary analysis and subset analysis of DCB type. BMS, bare metal stent; DCB, drug-coated balloon; DES, drug-eluting stent; PTA, percutaneous transluminal balloon angioplasty; TLR, target lesion revascularisation.

of around $£ 4000-£ 4500$ per QALY gained. Considering that clinical results of individual DCB have been found to differ significantly on the basis of different balloon pharmacokinetics and paclitaxel bioavailability, ${ }^{15}{ }^{16}$ we explored the potential effect of different DCB catheters. Our DCB subgroup analysis revealed that a $3.5 \mu \mathrm{g} / \mathrm{mm}^{2}$ paclitaxel balloon with a urea-based excipient was associated with the lowest rate of TLR totalling $11.2 \%$ at 24 months and a calculated cost impact of $£ 33$ per patient, a NNT of 4 and a most favourable ICER of around £2300 per QALY gained.

While the current NICE guidance ${ }^{3}$ does not consider DCB or DES as the first-line treatment in the SFA, the authors suggest that a subsequent revised version should carefully consider the aforementioned options, based on the presented robust clinical data and favourable health economic analysis. The economic impact of the different SFA treatments is based on the higher initial treatment costs versus the current standard of care, and the subsequent differential of the avoided or delayed TLRs - a classic 'spend now to save later' scenario. Costs per procedures avoided, based on the inverse of the absolute risk difference (or NNT), seem to provide very reasonable value not just for the healthcare system but also for the patients who can be spared repeat invasive procedures and may enjoy improved quality of life. We have identified very small NNT (range 4-11) in all of the explored scenarios of the base case and sensitivity analyses confirming the high efficacy of DES and DCB as first-line SFA therapies. Arguably, DES and DCB are not only protecting patients from recurrent symptomatic disease that may mandate reinterventions, but are also saving them from the inherent risk of potential complications, anxiety and inconvenience of having to undergo a repeat procedure.

To account for variable SFA lesion complexity, we expanded our analysis to include 1.5 drug-eluting devices on average. As expected, the latter approach showed an increase of incremental cost impact of around $£ 280-£ 300$ per patient at 24 months with a substantial increase of the corresponding ICER values. On the other hand, the results of our health economic decision analysis were sensitive in magnitude and direction to the actual list prices of the devices. Notably, a $10 \%$ price reduction raised both DES and DCB as dominant healthcare technologies by saving money for the NHS budget, while offering superior clinical outcomes. Hence, considering the fiercely competitive open market and ongoing pressure for cost savings in the current economic challenges facing the NHS, this analysis shows that drug-eluting endovascular technologies are potentially a new paradigm shift in the treatment of femoropopliteal disease.

The literature on economic analyses of DESs or DCBs is sparse to date. While there is a recent British cost-utility analysis on DCBs for infrainguinal disease ${ }^{1}$ projecting both lower costs and higher QALYs (ie, DCB was found dominant) for intermittent claudication and CLI, the analysis does not take newer studies into consideration. A Swiss cost-effectiveness study from $2013^{48}$ revealed that a DCB strategy, compared with PTA, is likewise economically dominant; however, that study was only based on clinical input parameters from one study. ${ }^{17}$ Three of the authors of the current study participated in a prior study investigating budget impact of endovascular strategies in the German and US healthcare systems. ${ }^{6}$ That study, based on clinical data available up to early 2013, found drug-eluting therapies to be associated with cost savings in both healthcare systems, when compared with PTA and BMS over a 2-year horizon.

In the present economic analysis, we performed an in-depth synthesis of numerous studies in the femoropopliteal segment. We first explored the options of DES or DCB as first-line treatments (primary analysis) and then also as secondary treatments during potential 


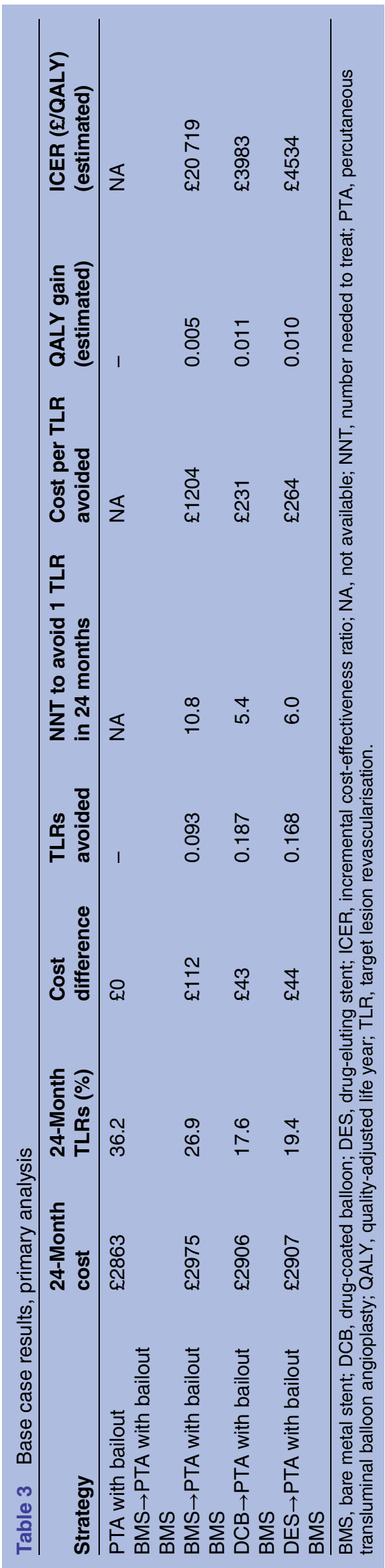

revascularisation to account for the wide variation of real-life clinical practice in the UK (secondary analysis). In the latter analysis, incremental cost per patient increased modestly (range £162-£259) compared with the primary analysis base case (range £43-£44). However, we consider those economic estimates as quite conservative because the present study considered a time horizon of only 2 years; hence, our model did not allow enough time for the expected clinical benefit of secondary drug-eluting treatments to materialise. Still, we note that the DCB with urea-based excipient produced more favourable clinical and economic results under all scenarios and conditions (see online supplementary appendix).

There are several limitations to this health economic study. First, we pursued a decision-analytic budget impact model, albeit based on true study data from a number of studies and including various competing balloon and stent endovascular treatments. Second, the index procedure in this study pertains to de novo lesions; however, we felt that this was the area with the greatest unmet clinical need, practice patterns are likely to vary in restenosis, and much less published evidence is currently available for restenoses. Third, our analysis was limited to a 2-year time horizon, and the ICER projections were based on simplified computer simulations under certain health utility assumptions. We speculate that a time horizon of at least 5 years would be more applicable for the femoropopliteal segment and ideally a cost-effectiveness study would take place within the context of a prospective randomised controlled trial. Notably, promising 5-year results have been recently released for a paclitaxel-eluting stent ${ }^{49}$ and a paclitaxelcoated balloon ${ }^{50}$ in the femoropopliteal segment. In addition, a quality of life and cost-effectiveness analysis of the randomised IN.PACT SFA trial at 2 years has been recently released from the perspective of the US healthcare system. ${ }^{51}$ The health economic findings from the IN.PACT SFA randomised trial are in line with the present results from the NHS perspective in the UK and also corroborate the fact that a $3.5 \mu \mathrm{g} / \mathrm{mm}^{2}$ urea excipient-based DCB may be a dominant femoropopliteal treatment option. ${ }^{51}$ Fourth, most of the currently available evidence was derived from outside of the UK. While disease biology and risk factors might be similar in other countries, the timing and type of intervention might lead to effect modification in terms of the lesions at the time of endovascular therapy. Last but not least, the synthesised evidence mostly applied for intermittent claudication and the population of CLI was underrepresented, resembling only $15-20 \%$ of enrolled participants in the included studies. Still, a recent meta-analysis and metaregression study of all available randomised trials investigating different DCB in the femoropopliteal segment has identified only a weak adverse relationship between incidence of CLI and reduction of TLR rates by paclitaxel-coated balloonson the other hand, paclitaxel dose was the strongest, 
Table 4 Base case results, secondary analysis

\begin{tabular}{|c|c|c|c|c|c|c|c|c|}
\hline Strategy & $\begin{array}{l}\text { 24-Month } \\
\text { cost }\end{array}$ & $\begin{array}{l}\text { 24-Month } \\
\text { TLRs (\%) }\end{array}$ & $\begin{array}{l}\text { Cost } \\
\text { difference }\end{array}$ & $\begin{array}{l}\text { TLRs } \\
\text { avoided }\end{array}$ & $\begin{array}{l}\text { NNT to avoid } 1 \text { TLR } \\
\text { in } 24 \text { months }\end{array}$ & $\begin{array}{l}\text { Cost per TLR } \\
\text { avoided }\end{array}$ & $\begin{array}{l}\text { QALY gain } \\
\text { (estimated) }\end{array}$ & $\begin{array}{l}\text { ICER (£/QALY) } \\
\text { (estimated) }\end{array}$ \\
\hline $\begin{array}{l}\text { PTA with bailout } \\
\text { BMS } \rightarrow \text { PTA with bailout } \\
\text { BMS }\end{array}$ & $£ 2863$ & 36.2 & $£ 0$ & - & NA & NA & - & NA \\
\hline $\begin{array}{l}\text { PTA with bailout } \\
\text { BMS } \rightarrow \text { DCB/DES } 50 \% \text { split }\end{array}$ & $£ 3014$ & 36.2 & $£ 151$ & - & NA & NA & - & NA \\
\hline $\mathrm{DCB} \rightarrow \mathrm{PTA}$ & $£ 2893$ & 17.6 & $£ 30$ & 0.187 & 5.4 & $£ 159$ & 0.011 & $£ 2739$ \\
\hline $\mathrm{DCB} \rightarrow \mathrm{DCB}$ & $£ 2983$ & 17.6 & $£ 120$ & 0.187 & 5.4 & $£ 641$ & 0.011 & $£ 11033$ \\
\hline $\begin{array}{l}\text { DCB with bailout } \\
\text { BMS } \rightarrow \text { PTA with bailout } \\
\text { BMS }\end{array}$ & $£ 3022$ & 19.4 & $£ 159$ & 0.168 & 6.0 & $£ 949$ & 0.010 & $£ 16335$ \\
\hline $\begin{array}{l}\text { DCB with bailout } \\
\text { BMS } \rightarrow \text { DCB with bailout } \\
\text { BMS }\end{array}$ & $£ 3122$ & 19.4 & $£ 259$ & 0.168 & 6.0 & $£ 1543$ & 0.010 & $£ 26538$ \\
\hline $\begin{array}{l}\text { DCB with bailout } \\
\text { BMS } \rightarrow \text { DCB/DES } 50 \% \text { split }\end{array}$ & $£ 3103$ & 19.4 & $£ 240$ & 0.168 & 6.0 & $£ 1432$ & 0.010 & $£ 24629$ \\
\hline DES $\rightarrow$ PTA & $£ 2892$ & 19.4 & $£ 29$ & 0.168 & 6.0 & $£ 175$ & 0.010 & $£ 3005$ \\
\hline $\mathrm{DES} \rightarrow \mathrm{DCB}$ & $£ 2992$ & 19.4 & $£ 129$ & 0.168 & 6.0 & $£ 767$ & 0.010 & $£ 13194$ \\
\hline $\mathrm{BMS} \rightarrow \mathrm{PTA}$ & $£ 2954$ & 26.9 & $£ 91$ & 0.093 & 10.8 & $£ 982$ & 0.005 & $£ 16890$ \\
\hline $\mathrm{BMS} \rightarrow \mathrm{DCB}$ & $£ 3092$ & 26.9 & $£ 229$ & 0.093 & 10.8 & $£ 2465$ & 0.005 & $£ 42413$ \\
\hline
\end{tabular}

BMS, bare metal stent; DCB, drug-coated balloon; DES, drug-eluting stent; ICER, incremental cost-effectiveness ratio; NA, not available; NNT, number needed to treat; PTA, percutaneous transluminal balloon angioplasty; QALY, quality-adjusted life year; TLR, target lesion revascularisation. 
highly significant predictor of the expected biological effect size of DCB treatment. ${ }^{16}$ Hence, the authors consider the present results generalisable for different treatment indications for femoropopliteal disease.

In conclusion, the widespread adoption of drug-eluting endovascular therapies for femoropopliteal disease would add meaningful clinical benefit at reasonable additional costs to the NHS and should be carefully considered in future revised guidelines. Based on currently available data, both DES and DCB offer the highest clinical and economic value compared with uncoated balloon angioplasty and/or BMS. Contrary to DES, DCB leave no permanent metal implant behind and-in particular with the use of a $3.5 \mu \mathrm{g} / \mathrm{mm}^{2}$ ureabased DCB — might be associated with added clinical benefit and a potentially more favourable economic profile. More randomised trials with an a priori clinical effectiveness and cost-effectiveness design, especially in the case of CLI, are warranted.

\section{Twitter Follow Konstantinos Katsanos at @DrKKatsanos}

Contributors The study was initiated by JBP. All authors were involved in the design of the study. The systematic literature review and its update were performed by JBP, AMG and BPG. The clinical and economic analyses were performed by JBP and BPG. The manuscript was written by KK, JBP and BPG, with the other authors providing critical review and editing. All authors have approved the final submitted version of the manuscript.

Funding Wing Tech Inc provided health economic consulting services to Medtronic.

Competing interests For the purposes of this project, $\mathrm{KK}, \mathrm{HZ}$ and $\mathrm{TC}$ served as unpaid scientific advisors to Medtronic, a manufacturer of angioplasty balloons and stents. Wing Tech Inc. (JBP, BPG, AMG) provides healtheconomic consulting services to Medtronic.

Provenance and peer review Not commissioned; externally peer reviewed.

Data sharing statement No additional data are available.

Open Access This is an Open Access article distributed in accordance with the Creative Commons Attribution Non Commercial (CC BY-NC 4.0) license, which permits others to distribute, remix, adapt, build upon this work noncommercially, and license their derivative works on different terms, provided the original work is properly cited and the use is non-commercial. See: http:// creativecommons.org/licenses/by-nc/4.0/

\section{REFERENCES}

1. Kearns BC, Michaels JA, Stevenson MD, et al. Cost-effectiveness analysis of enhancements to angioplasty for infrainguinal arterial disease. Br J Surg 2013;100:1180-8.

2. Fowkes FGR, Rudan D, Rudan I, et al. Comparison of global estimates of prevalence and risk factors for peripheral artery disease in 2000 and 2010: a systematic review and analysis. Lancet 2013;382:1329-40.

3. National Institute for Health and Care Excellence. Peripheral arterial disease: diagnosis and management. 2012. https://www.nice.org.uk guidance/cg147

4. Bosiers M, Torsello G, Gissler HM, et al. Nitinol stent implantation in long superficial femoral artery lesions: 12-month results of the DURABILITY I study. J Endovasc Ther 2009;16:261-9.

5. Katsanos K, Spiliopoulos S, Karunanithy N, et al. Bayesian network meta-analysis of nitinol stents, covered stents, drug-eluting stents, and drug-coated balloons in the femoropopliteal artery. $J$ Vasc Surg 2014;59:1123-33.e8.

6. Pietzsch JB, Geisler BP, Garner AM, et al. Economic analysis of endovascular interventions for femoropopliteal arterial disease: a systematic review and budget impact model for the United States and Germany. Catheter Cardiovasc Interv 2014;84:546-54.

7. Laird JR, Schneider PA, Tepe G, et al. Durability of treatment effect using a drug-coated balloon for femoropopliteal lesions: 24-month results of IN.PACT SFA. J Am Coll Cardiol 2015;66:2329-38.

8. Laird JR, Katzen BT, Scheinert D, et al. Nitinol stent implantation versus balloon angioplasty for lesions in the superficial femoral artery and proximal popliteal artery: twelve-month results from the RESILIENT randomized trial. Circ Cardiovasc Interv 2010;3:267-76.

9. Laird JR, Jain A, Zeller T, et al. Nitinol stent implantation in the superficial femoral artery and proximal popliteal artery: twelve-month results from the complete SE multicenter trial. J Endovasc Ther 2014;21:202-12.

10. Litsky J, Chanda A, Stilp E, et al. Critical evaluation of stents in the peripheral arterial disease of the superficial femoral artery-focus on the paclitaxel eluting stent. Med Devices (Auckl) 2014;7:149-56.

11. Tepe G, Laird J, Schneider P, et al. Drug-coated balloon versus standard percutaneous transluminal angioplasty for the treatment of superficial femoral and popliteal peripheral artery disease: 12-month results from the IN.PACT SFA randomized trial. Circulation 2015;131:495-502.

12. Schillinger $M$, Sabeti $S$, Loewe $C$, et al. Balloon angioplasty versus implantation of nitinol stents in the superficial femoral artery. $N$ Engl J Med 2006;354:1879-88.

13. Chalmers N, Walker PT, Belli AM, et al. Randomized trial of the SMART stent versus balloon angioplasty in long superficial femora artery lesions: the SUPER study. Cardiovasc Intervent Radiol 2013;36:353-61.

14. Gongora CA, Shibuya M, Wessler JD, et al. Impact of paclitaxel dose on tissue pharmacokinetics and vascular healing: a comparative drug-coated balloon study in the familial hypercholesterolemic swine model of superficial femoral in-stent restenosis. JACC Cardiovasc Interv 2015;8:1115-23.

15. Granada JF, Stenoien M, Buszman PP, et al. Mechanisms of tissue uptake and retention of paclitaxel-coated balloons: impact on neointimal proliferation and healing. Open Heart 2014;1:e000117.

16. Katsanos K, Spiliopoulos S, Paraskevopoulos I, et al. Systematic review and meta-analysis of randomized controlled trials of paclitaxel-coated balloon angioplasty in the femoropopliteal artery: role of paclitaxel dose and bioavailability. J Endovasc Ther 2016;23:356-70.

17. Tepe $\mathrm{G}$, Zeller $\mathrm{T}$, Albrecht $\mathrm{T}$, et al. Local delivery of paclitaxel to inhibit restenosis during angioplasty of the leg. $N$ Engl J Med 2008;358:689-99.

18. Werk M, Langner S, Reinkensmeier B, et al. Inhibition of restenosis in femoropopliteal arteries: paclitaxel-coated versus uncoated balloon: femoral paclitaxel randomized pilot trial. Circulation 2008;118:1358-65

19. Laird JR, Katzen BT, Scheinert D, et al. Nitinol stent implantation vs. balloon angioplasty for lesions in the superficial femoral and proximal popliteal arteries of patients with claudication: three-year follow-up from the RESILIENT randomized trial. J Endovasc Ther 2012:19:1-9.

20. Krankenberg $H$, Schlüter $M$, Steinkamp HJ, et al. Nitinol stent implantation versus percutaneous transluminal angioplasty in superficial femoral artery lesions up to $10 \mathrm{~cm}$ in length: the femora artery stenting trial (FAST). Circulation 2007;116:285-92.

21. Dake MD, Ansel GM, Jaff MR, et al. Paclitaxel-eluting stents show superiority to balloon angioplasty and bare metal stents in femoropopliteal disease: twelve-month Zilver PTX randomized study results. Circ Cardiovasc Interv 2011;4:495-504.

22. Dake MD, Ansel GM, Jaff MR, et al. Sustained safety and effectiveness of paclitaxel-eluting stents for femoropopliteal lesions: 2-year follow-up from the Zilver PTX randomized and single-arm clinical studies. J Am Coll Cardiol 2013;61:2417-27.

23. Werk M, Albrecht T, Meyer DR, et al. Paclitaxel-coated balloons reduce restenosis after femoro-popliteal angioplasty: evidence from the randomized PACIFIER trial. Circ Cardiovasc Interv 2012:5:831-40.

24. Scheinert D, Schulte KL, Zeller T, et al. Paclitaxel-releasing balloon in femoropopliteal lesions using a BTHC excipient: twelve-month results from the BIOLUX $\mathrm{P}-\mathrm{I}$ randomized trial. J Endovasc Ther 2015;22:14-21.

25. Scheinert D, Duda S, Zeller T, et al. The LEVANT I (Lutonix paclitaxel-coated balloon for the prevention of femoropoplitea restenosis) trial for femoropopliteal revascularization: first-in-human randomized trial of low-dose drug-coated balloon versus uncoated balloon angioplasty. JACC Cardiovasc Interv 2014;7:10-19.

26. Rosenfield K, Jaff MR, White CJ, et al. Trial of a paclitaxel-coated balloon for femoropopliteal artery disease. $N$ Engl J Med 2015;373:145-53. 
27. Dattilo R, Himmelstein SI, Cuff RF. The COMPLIANCE 360 degrees Trial: a randomized, prospective, multicenter, pilot study comparing acute and long-term results of orbital atherectomy to balloon angioplasty for calcified femoropopliteal disease. J Invasive Cardiol 2014;26:355-60.

28. Duda SH, Bosiers M, Lammer J, et al. Drug-eluting and bare nitinol stents for the treatment of atherosclerotic lesions in the superficial femoral artery: long-term results from the SIROCCO trial. $J$ Endovasc Ther 2006;13:701-10.

29. Diehl SJ, Gerblich F, Jochum S, et al. Twelve-month results of the EverFlex stent in the superficial femoral artery. $J$ Vasc Interv Radiol 2012;23:1317-22.

30. Banerjee S, Das TS, Abu-Fadel MS, et al. Pilot trial of cryoplasty or conventional balloon post-dilation of nitinol stents for revascularization of peripheral arterial segments: the COBRA trial. J Am Coll Cardiol 2012;60:1352-9.

31. Kralj I, Boos I, Müller-Bühl U. Three year results of endovascular therapy with a new generation nitinol stent for femoro-popliteal artery lesions-a single-center outcome analysis of a subcohort of MISAGO 2 study. Vasa 2013;42:340-9.

32. Rastan A, Krankenberg $\mathrm{H}$, Baumgartner I, et al. Stent placement versus balloon angioplasty for the treatment of obstructive lesions of the popliteal artery: a prospective, multicenter, randomized trial. Circulation 2013;127:2535-41.

33. Tadros RO, Faries PL, Rocha-Singh KJ, et al. The impact of sex on angioplasty and primary stenting for femoropopliteal occlusive disease: results of the DURABILITY II trial. Ann Vasc Surg 2014;28:1-9.

34. Werner M, Piorkowski M, Thieme $\mathrm{M}$, et al. SUMMIT registry: one-year outcomes after implantation of the EPIC self-expanding nitinol stent in the femoropopliteal segment. $J$ Endovasc Ther 2013;20:759-66.

35. Gabrielli R, Rosati MS, Chiappa R, et al. First clinical experience with the Innova versus the Protege EverFlex self-expanding bare metal stents in superficial femoral artery occlusions. Thorac Cardiovasc Surg 2015;63:158-63.

36. Garcia L, Jaff MR, Metzger C, et al. Wire-interwoven nitinol stent outcome in the superficial femoral and proximal popliteal arteries: twelve-month results of the SUPERB trial. Circ Cardiovasc Interv 2015;8:e000937.

37. Krankenberg $\mathrm{H}$, Tübler T, Sixt $\mathrm{S}$, et al. German multicenter real-world registry of stenting for superficial femoral artery disease: clinical results and predictive factors for revascularization. $J$ Endovasc Ther 2014;21:463-71.

38. Werner M, Paetzold A, Banning-Eichenseer U, et al. Treatment of complex atherosclerotic femoropopliteal artery disease with a self-expanding interwoven nitinol stent: midterm results from the Leipzig SUPERA 500 registry. Eurolntervention 2014;10:861-8.

39. Micari A, Cioppa A, Vadalà G, et al. Clinical evaluation of a paclitaxel-eluting balloon for treatment of femoropopliteal arterial disease: 12-month results from a multicenter Italian registry. JACC Cardiovasc Interv 2012;5:331-8.

40. Micari A, Cioppa A, Vadalà $G$, et al. 2-year results of paclitaxel-eluting balloons for femoropopliteal artery disease: evidence from a multicenter registry. JACC Cardiovasc Interv 2013;6:282-9.

41. Schroeder H, Meyer DR, Lux B, et al. Two-year results of a low-dose drug-coated balloon for revascularization of the femoropopliteal artery: Outcomes from the ILLUMENATE first-in-human study. Catheter Cardiovasc Interv 2015;86:278-86.

42. Dake MD, Ansel GM, Jaff MR, et al. Sustained safety and effectiveness of paclitaxel-eluting stents for femoropopliteal lesions: 2-year follow-up from the Zilver PTX randomized and single-arm clinical studies. J Am Coll Cardiol 2013;61:2417-27.

43. Lammer J, Bosiers M, Zeller T, et al. First clinical trial of nitinol self-expanding everolimus-eluting stent implantation for peripheral arterial occlusive disease. J Vasc Surg 2011;54:394-401.

44. Cortese B, Granada JF, Scheller B, et al. Drug-coated balloon treatment for lower extremity vascular disease intervention: an international positioning documentdagger. Eur Heart $J$ 2016;37:1096-103

45. Katsanos K, Tepe G, Tsetis D, et al. Standards of practice for superficial femoral and popliteal artery angioplasty and stenting. Cardiovasc Intervent Radiol 2014;37:592-603.

46. Diamantopoulos A, Katsanos K. Treating femoropopliteal disease: established and emerging technologies. Semin Intervent Radiol 2014;31:345-52.

47. Pietzsch JB, Weber SA, Pietzsch ML, et al. The impact of new endovascular therapies for femoropopliteal arterial disease on therapy utilization and case volumes in Germany, 2009-2013. Value Health 2015;18:A366.

48. Diehm N, Schneider H. Cost-effectiveness analysis of paclitaxel-coated balloons for endovascular therapy of femoropopliteal arterial obstructions. J Endovasc Ther 2013;20:819-25

49. Dake MD. The Zilver PTX randomized trial of treating femoropopliteal artery disease: 5-year results. Las Veags, NV, USA Vascular Interventional Advances (VIVA), 2014.

50. Tepe G, Schnorr B, Albrecht T, et al. Angioplasty of femoral-popliteal arteries with drug-coated balloons: 5-year follow-up of the THUNDER trial. JACC Cardiovasc Interv 2015;8(Pt A):102-8.

51. Cohen DJ. IN.PACT SF a health-economic study. Las Vegas, NV, USA: VIVA, 2015 